\title{
On Functions with the Cauchy Difference Bounded by a Functional
} by

\section{Włodzimierz FECHNER}

Presented by Andrzej LASOTA

Summary. K. Baron and Z. Kominek [2] have studied the functional inequality

$$
f(x+y)-f(x)-f(y) \geq \phi(x, y), \quad x, y \in X,
$$

under the assumptions that $X$ is a real linear space, $\phi$ is homogeneous with respect to the second variable and $f$ satisfies certain regularity conditions. In particular, they have shown that $\phi$ is bilinear and symmetric and $f$ has a representation of the form $f(x)=\frac{1}{2} \phi(x, x)+L(x)$ for $x \in X$, where $L$ is a linear function.

The purpose of the present paper is to consider this functional inequality under different assumptions upon $X, f$ and $\phi$. In particular we will give conditions which force biadditivity and symmetry of $\phi$ and the representation $f(x)=\frac{1}{2} \phi(x, x)-A(x)$ for $x \in X$, where $A$ is a subadditive function.

Let $(X,+)$ be an abelian group. We consider the functional inequality

$$
f(x+y)-f(x)-f(y) \geq \phi(x, y), \quad x, y \in X,
$$

where $\phi: X \times X \rightarrow \mathbb{R}$ and $f: X \rightarrow \mathbb{R}$ are unknown mappings.

It is easy to check that if $\phi: X \times X \rightarrow \mathbb{R}$ is biadditive and symmetric, $A: X \rightarrow \mathbb{R}$ is subadditive and $f: X \rightarrow \mathbb{R}$ is defined by the formula $f(x):=$ $\frac{1}{2} \phi(x, x)-A(x)$ for $x \in X$, then (1) holds. We are going to provide conditions under which the converse implication is valid.

Proposition. If $f: X \rightarrow \mathbb{R}$ and $\phi: X \times X \rightarrow \mathbb{R}$ satisfy (1) and

$$
\phi(x,-x) \geq-\phi(x, x), \quad x \in X,
$$

then: (a) $f(0) \leq 0$; (b) $f(x)+f(-x) \leq \phi(x, x)$ for $x \in X$; (c) $f(2 x) \geq$ $3 f(x)+f(-x)$ for $x \in X$.

2000 Mathematics Subject Classification: Primary 39B62, 39B72.

Key words and phrases: functional inequality; subadditive, quadratic and biadditive functionals. 
Proof. The assumption (2) implies that $\phi(0,0) \geq 0$; thus applying (1) with $x=0$ and $y=0$ we get $f(0) \leq 0$. Using this and substituting $y:=-x$ in (1) we derive (b). Substituting $y:=x$ in (1) and using (b) proves (c). This completes the proof.

In what follows we make use of a result of Karol Baron (see S. Rolewicz [4, Lemma 5.7]). A careful inspection of the original proof allows us to weaken certain assumptions of this lemma. The original result reads as follows.

Lemma (K. Baron). Assume that $f: X \rightarrow \mathbb{R}$ and $\phi: X \times X \rightarrow \mathbb{R}$ satisfy (1). If $f$ is even, $f(2 x)=4 f(x)$ and $\phi(x, \cdot)$ is odd for every $x \in X$, then there exists a biadditive and symmetric functional $B: X \times X \rightarrow \mathbb{R}$ such that $\phi=2 B$ and $f(x)=B(x, x)$ for every $x \in X$.

We have the following modification of this lemma.

Lemma 1. Assume that $f: X \rightarrow \mathbb{R}$ and $\phi: X \times X \rightarrow \mathbb{R}$ satisfy (1). If

$$
\phi(x,-y) \geq-\phi(x, y), \quad x, y \in X,
$$

and

$$
f(2 x) \leq 4 f(x), \quad x \in X,
$$

then

$$
f(x)=\frac{1}{2} \phi(x, x), \quad x \in X .
$$

Moreover, $\phi$ is biadditive and symmetric.

Proof. Using the inequality (c) of the Proposition and (4) we see that $f(x) \geq f(-x)$ for $x \in X$, which proves that $f$ is even. Setting $-y$ instead of $y$ in (1) we obtain

$$
f(x-y)-f(x)-f(-y) \geq \phi(x,-y) \geq-\phi(x, y), \quad x, y \in X .
$$

Adding this to (1) and using the evenness of $f$ leads to

$$
f(x+y)+f(x-y) \geq 2 f(x)+2 f(y), \quad x, y \in X .
$$

Fix $u, v \in X$. Applying the above inequality with $x=u+v$ and $y=u-v$ we infer that

$$
4 f(u)+4 f(v) \geq f(2 u)+f(2 v) \geq 2 f(u+v)+2 f(u-v), \quad u, v \in X .
$$

Therefore $f$ is a quadratic function, i.e.

$$
f(x+y)+f(x-y)=2 f(x)+2 f(y), \quad x, y \in X .
$$

So, there exists a biadditive and symmetric functional $B: X \times X \rightarrow \mathbb{R}$ such that $f(x)=B(x, x)$ for $x \in X$ (see e.g. J. Aczél \& J. Dhombres [1, Chapter 11, Proposition 1]). It is easy to check that

$$
B(x, y)=\frac{1}{2}[f(x+y)-f(x)-f(y)], \quad x, y \in X .
$$


Now, assumption (3) and the biadditivity of $B$ imply that $2 B=\phi$. This completes the proof.

TheOrem 1. Assume that $f: X \rightarrow \mathbb{R}$ and $\phi: X \times X \rightarrow \mathbb{R}$ satisfy (1), (3) and

$$
\begin{array}{ll}
\limsup _{n \rightarrow \infty} \frac{1}{4^{n}} \phi\left(2^{n} x, 2^{n} x\right)<\infty, & x \in X, \\
\liminf _{n \rightarrow \infty} \frac{1}{4^{n}} \phi\left(2^{n} x, 2^{n} y\right) \geq \phi(x, y), & x, y \in X .
\end{array}
$$

If $f$ is even, then there exists a subadditive function $A: X \rightarrow \mathbb{R}$ such that

$$
f(x)=\frac{1}{2} \phi(x, x)-A(x), \quad x \in X .
$$

Moreover, $\phi$ is biadditive and symmetric.

Proof. Fix an $x \in X$ and a positive integer $n$. By the evenness of $f$ and the Proposition we get

$$
\frac{1}{4^{n-1}} f\left(2^{n-1} x\right) \leq \frac{1}{4^{n}} f\left(2^{n} x\right) \leq \frac{1}{4^{n}} \cdot \frac{1}{2} \phi\left(2^{n} x, 2^{n} x\right) .
$$

The first part of the assumption (5) implies that the right-hand side of this inequality is bounded by a real constant which does not depend on $n$. Therefore the formula

$$
Q(x):=\lim _{n \rightarrow+\infty} \frac{1}{4^{n}} f\left(2^{n} x\right), \quad x \in X,
$$

correctly defines a map $Q: X \rightarrow \mathbb{R}$. Moreover, $Q(2 x)=4 Q(x)$ for $x \in X$ and the following inequality is satisfied:

$$
\begin{aligned}
Q(x+y)-Q(x)-Q(y) & =\lim _{n \rightarrow \infty}\left[\frac{1}{4^{n}} f\left(2^{n} x+2^{n} y\right)-\frac{1}{4^{n}} f\left(2^{n} x\right)-\frac{1}{4^{n}} f\left(2^{n} y\right)\right] \\
& \geq \liminf _{n \rightarrow \infty} \frac{1}{4^{n}} \phi\left(2^{n} x, 2^{n} y\right) \geq \phi(x, y), \quad x, y \in X .
\end{aligned}
$$

Lemma 1 states that $\phi$ is biadditive and symmetric and $Q(x)=\frac{1}{2} \phi(x, x)$ for $x \in X$. In particular $Q(x+y)-Q(x)-Q(y)=\phi(x, y)$ for $x, y \in X$. From this and (1), it is easy to check that $A:=Q-f$ is subadditive. This completes the proof.

Corollary 1. Assume that $f: X \rightarrow \mathbb{R}$ and $\phi: X \times X \rightarrow \mathbb{R}$ satisfy (1), (3), (5) and

$$
\phi(-x,-y)=\phi(x, y), \quad x, y \in X .
$$

Then there exists a subadditive function $A: X \rightarrow \mathbb{R}$ such that

$$
f(x)=\frac{1}{2} \phi(x, x)-A(x), \quad x \in X .
$$

Moreover, $\phi$ is biadditive and symmetric. 
Proof. Define $h: X \rightarrow \mathbb{R}$ by $h(x):=\frac{1}{2}(f(x)+f(-x))$ for $x \in X$. Assumption (6) implies that

$$
h(x+y)-h(x)-h(y) \geq \phi(x, y), \quad x, y \in X .
$$

Using Theorem 1 with $f$ replaced by $h$ we get the biadditivity and symmetry of $\phi$. Now, one may easily check that the map $A: X \rightarrow \mathbb{R}$ given by $A(x):=$ $\frac{1}{2} \phi(x, x)-f(x)$ for $x \in X$ is subadditive. This completes the proof.

Now, we are going to provide conditions which, in particular, allow us to omit the assumption (6) and to weaken (5). We start with a lemma.

Lemma 2. If $f: X \rightarrow \mathbb{R}$ and $\phi: X \times X \rightarrow \mathbb{R}$ satisfy (1), (2) and $f$ is odd then $f(2 x)=2 f(x)$ and $\phi(x, x)=0$ for $x \in X$. Moreover, if $\phi$ satisfies (3), then $f$ is additive and $\phi=0$.

Proof. Fix an $x \in X$. Since $f$ is odd, we get

$$
\begin{aligned}
f(2 x)-2 f(x) & =-[f(-2 x)-2 f(-x)] \leq-\phi(-x,-x) \leq \phi(-x, x) \\
& \leq f(-x+x)-f(-x)-f(x)=0,
\end{aligned}
$$

whence, again by the oddness of $f$, we obtain $f(2 x)=2 f(x)$ for $x \in X$ and, in consequence, $\phi(x, x)=0$ for $x \in X$.

Now, assume (3) and let $x, y \in X$. Using the assumption (3) and (1) twice we obtain

$f(x-y)-f(x)-f(-y) \geq \phi(x,-y) \geq-\phi(x, y) \geq-f(x+y)+f(x)+f(y)$,

which means that

$$
f(x+y)+f(x-y) \geq 2 f(x) .
$$

Interchanging the roles of $x$ and $y$ we obtain

$$
f(y+x)+f(y-x) \geq 2 f(y) .
$$

Summing up these two inequalities we derive the superadditivity of $f$, which together with its oddness implies that $f$ is additive and $\phi \leq 0$. Using this and (3) we finally get $\phi=0$. This completes the proof.

The following lemma provides sufficient conditions for the function $f$ to satisfy the assumption (4).

Recall that a group $X$ is called uniquely 2-divisible if the map $X \ni x \mapsto$ $x+x \in X$ is bijective.

Lemma 3. Assume that $X$ is uniquely 2-divisible, $f: X \rightarrow \mathbb{R}$ and $\phi: X \times$ $X \rightarrow \mathbb{R}$ satisfy (1), (2) and

$$
\phi(2 x, 2 x) \leq 4 \phi(x, x), \quad x \in X .
$$

If $f$ is nonnegative and even, then $f(x)=\frac{1}{2} \phi(x, x)$ for $x \in X$. 
Proof. By the Proposition, for every $x \in X$ and every positive integer $n$ we have $4^{n} f\left(x / 2^{n}\right) \geq 4^{n+1} f\left(x / 2^{n+1}\right) \geq 0$. So, the sequence $\left(4^{n} f\left(x / 2^{n}\right)\right)_{n \in \mathbb{N}}$ is pointwise convergent. In particular, $\lim _{n \rightarrow \infty} 2^{n} f\left(x / 2^{n}\right)=0$ for every $x \in X$.

Now, fix an $x \in X$. Using (1) and (7), by induction, we get

$$
2^{k} f\left(\frac{x}{2^{k-1}}\right)-2^{k+1} f\left(\frac{x}{2^{k}}\right) \geq 2^{k} \phi\left(\frac{x}{2^{k}}, \frac{x}{2^{k}}\right) \geq \frac{1}{2^{k}} \phi(x, x)
$$

for all $k \in \mathbb{N}$. Summing up these inequalities for $k \in\{1, \ldots, n\}$ we get

$$
2 f(x)-2^{n+1} f\left(\frac{x}{2^{n}}\right) \geq \sum_{k=1}^{n} \frac{1}{2^{k}} \phi(x, x), \quad n \in \mathbb{N} .
$$

Letting $n$ tend to $+\infty$ yields $2 f(x) \geq \phi(x, x)$. Since the Proposition provides the opposite inequality, the proof is complete.

The following result yields an analogue of Corollary 1 in the paper [2] of K. Baron and Z. Kominek.

Theorem 2. Assume $X$ to be uniquely 2-divisible. If $f: X \rightarrow \mathbb{R}$ and $\phi: X \times X \rightarrow \mathbb{R}$ satisfy (1), (3), (7) and

$$
f(x)+f(-x) \geq 0, \quad x \in X,
$$

then there exists an additive function $a: X \rightarrow \mathbb{R}$ such that

$$
f(x)=\frac{1}{2} \phi(x, x)+a(x), \quad x \in X .
$$

Moreover, $\phi$ is biadditive and symmetric.

Proof. Define $h, a: X \rightarrow \mathbb{R}$ by $h(x):=\frac{1}{2}[f(x)+f(-x)]$ and $a(x):=$ $\frac{1}{2}[f(x)-f(-x)], x \in X$. Clearly $h$ is even whereas $a$ is odd. Next, define $\phi_{1}: X \times X \rightarrow \mathbb{R}$ by $\phi_{1}(x, y):=\frac{1}{2}[\phi(x, y)+\phi(-x,-y)]$ for $x, y \in X$. It is easy to check that $h$ and $\phi_{1}$ satisfy the assumptions of Lemma 3. So, $h(x)=\frac{1}{2} \phi_{1}(x, x)$ for $x \in X$. Now, observe that the assumptions of Lemma 1 are satisfied. Therefore $\phi_{1}$ is biadditive and symmetric and, in consequence,

$$
h(x+y)-h(x)-h(y)=\phi_{1}(x, y), \quad x, y \in X .
$$

Define $\phi_{2}: X \times X \rightarrow \mathbb{R}$ by $\phi_{2}:=\phi-\phi_{1}$. Note that $\phi_{2}(x,-y) \geq-\phi_{2}(x, y)$ for $x, y \in X$ and

$$
a(x+y)-a(x)-a(y) \geq \phi_{2}(x, y), \quad x, y \in X .
$$

Now, Lemma 2 applied for $f=a$ and $\phi=\phi_{2}$ states that $a$ is additive and $\phi_{2}=0$, i.e. $\phi=\phi_{1}$. This completes the proof.

A similar reasoning allows us to derive the following corollary from Lemmas 2 and 3 .

Corollary 2. Assume $X$ to be uniquely 2-divisible. If $f: X \rightarrow \mathbb{R}$ and $\phi: X \times X \rightarrow \mathbb{R}$ satisfy (1), (2), (7) and (8), then there exists an odd function 
$a: X \rightarrow \mathbb{R}$ such that $a(2 x)=2 a(x)$ for $x \in X$ and

$$
f(x)=\frac{1}{2} \phi(x, x)+a(x), \quad x \in X .
$$

Moreover, $\phi(2 x, 2 x)=4 \phi(x, x) \geq 0$ for $x \in X$.

Proof. Define $a, h, \phi_{1}$ and $\phi_{2}$ as in the previous proof. Lemma 3 implies that $h(x)=\frac{1}{2} \phi_{1}(x, x)$ and $h(2 x)=4 h(x)$ for $x \in X$. We are going to show that $\phi_{2}$ satisfies (2). Since $\phi_{2}=\phi-\phi_{1}$, it suffices to prove that $\phi_{1}(x,-x)=$ $-\phi_{1}(x, x)$ for $x \in X$. But

$$
\begin{aligned}
-2 h(x) & =h(-x+x)-h(-x)-h(x) \geq \phi_{1}(-x, x) \geq-\phi_{1}(-x,-x) \\
& =-2 h(x),
\end{aligned}
$$

which is what we wanted. Lemma 2 implies that $a(2 x)=2 a(x)$ and $\phi_{2}(x, x)$ $=0$, i.e. $h(x)=\frac{1}{2} \phi_{1}(x, x)=\frac{1}{2} \phi(x, x)$ for $x \in X$. This completes the proof.

We end this paper with some additional remarks.

Remark 1. If $c \in(0, \infty), f: \mathbb{R} \rightarrow \mathbb{R}$ is constant and equal to $-c$, $\phi: \mathbb{R} \times \mathbb{R} \rightarrow \mathbb{R}$ is constant and equal to $c$, then (1), (3) and (7) are satisfied. So, the assumption (4) in Lemma 1 cannot be omitted, the assumption (5) in Theorem 1 and Corollary 1 cannot be replaced by (7), the nonnegativity of $f$ in Lemma 3 cannot be replaced by its boundedness, and the assumption (8) in Theorem 2 cannot be omitted.

REMARK 2 . Let $\varphi: \mathbb{R} \rightarrow \mathbb{R}$ be a nonzero and even function which satisfies the equality

$$
\varphi(2 t)=2 \varphi(t), \quad t \in \mathbb{R}
$$

(see e.g. M. Kuczma, B. Choczewski and R. Ger [3] for examples of such functions). Define $f: \mathbb{R} \rightarrow \mathbb{R}$ and $\phi: \mathbb{R} \times \mathbb{R} \rightarrow \mathbb{R}$ by

$$
\begin{aligned}
f(x) & :=(\varphi(x))^{2}, \quad x \in \mathbb{R}, \\
\phi(x, y) & :=f(x+y)-f(x)-f(y), \quad x, y \in \mathbb{R} .
\end{aligned}
$$

Then inequality (1) is satisfied, and $f$ is even, nonnegative and satisfies (4). Moreover, $\phi$ satisfies (2), (5) and (6). So, in Lemma 1, Theorems 1 and 2 and Corollary 1, (3) cannot be replaced by (2).

Remark 3. Let $(X ;\|\cdot\|)$ be a normed linear space. Corollary 1 implies that the inequality

$$
f(x+y)-f(x)-f(y) \geq\|x\| \cdot\|y\|, \quad x, y \in X,
$$

has no solution. In fact, the function $\phi(x, y):=\|x\| \cdot\|y\|, x, y \in X$, satisfies (3), (5) and (6), but $\phi$ fails to be biadditive.

In this inequality $X$ may stand for an abelian group and the norm can be replaced by any real function, which is nonzero, nonnegative, even and 2-homogeneous. 


\section{References}

[1] J. Aczél and J. Dhombres, Functional Equations in Several Variables, Encyclopedia Math. Appl. 31, Cambridge Univ. Press, Cambridge, 1989.

[2] K. Baron and Z. Kominek, On functionals with the Cauchy difference bounded by a homogeneous functional, Bull. Polish Acad. Sci. Math. 51 (2003), 301-307.

[3] M. Kuczma, B. Choczewski and R. Ger, Iterative Functional Equations, Encyclopedia Math. Appl. 32, Cambridge Univ. Press, Cambridge, 1990.

[4] S. Rolewicz, $\Phi$-convex functions defined on metric spaces, Int. J. Math. Sci. 115 (2003), 2631-2652.

Włodzimierz Fechner

Institute of Mathematics

Silesian University

Bankowa 14

40-007 Katowice, Poland

E-mail: fechner@ux2.math.us.edu.pl

Received March 31, 2004;

received in final form August 3, 2004 